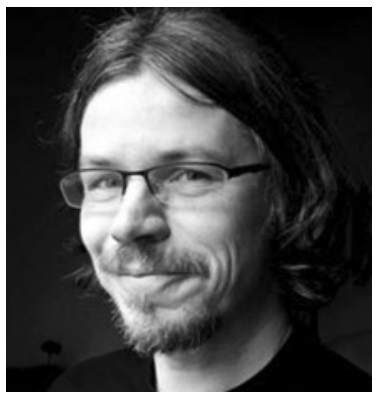

Peter Pagel

Chefredakteur

\title{
IT ist Vielfalt
}

Informationstechnologie hat enorm viele Facetten, deshalb ist es guter Brauch im Informatik Spektrum, neben Themenheften auch solche zu veröffentlichen, die Beiträge aus unterschiedlichen Bereichen zusammenbringen. Dieses Heft ist so eines.

Dass gute Softwarearchitektur betriebswirtschaftlich sinnvoll ist, zeigt der Aufsatz von Frank J. Furrer und Hendrik Schön. Dazu wählen die Autoren einen modellbasierten Ansatz. E. W. Udo Küppers macht sich in seinem Artikel Gedanken über die Entwicklung im Bereich der künstlichen Intelligenz. Erfahren Sie, was er mit „Programmierter Maschinendummheit" meint und welche Entwicklungen er im Zusammenspiel zwischen menschlicher und maschineller Intelligenz im Rahmen der digitalen Transformation auf uns zukommen sieht. Auch Arno Rolf beschäftigt sich mit der digitalen Transformation. Sein Fokus liegt darauf, was Informatiker über die digitale Transformation lernen sollten, um für ihre zukünftigen Aufgaben gerüstet zu sein. Neben einer anspruchsvollen Fachausbildung müssten Studierende mit der Komplexität der bevorstehenden digitalen Transformation vertraut gemacht werden, meint Rolf. Er präsentiert einen didaktischen Ansatz, der in Bachelorstudiengängen der Informatik und Wirtschaftsinformatik bereits umgesetzt wurde. Hans Pongratz, Geschäftsführender Vizepräsident IT-Systeme und Dienstleistungen (CIO) an der TU München berichtet in seinem Artikel von der praktischen Umsetzung der DSVGO an der Universität.

Außerdem haben wir diesmal ein ausführliches Interview mit Prof. Dr. Thomas Ludwig, der über seine Arbeit am Deutschen Klimarechenzentrum (DKRZ) in Hamburg berichtet und über seine Ideen für seine künftige Rolle als Hauptherausgeber des Informatik Spektrums. Das Gespräch führten der aktuelle Hauptherausgeber Prof. Dr. Arndt Bode und Chefredakteur Peter Pagel.

Schließlich befasst sich der Beitrag „Divide et impera" von H.-J. Burkhardt und Rainer Prinoth mit den Grenzen der Künstlichen Intelligenz. "Wenn es zur Postkutschenzeit, also schon vor weit mehr als hundert Jahren, die Kl-Methoden und die zugehörigen technischen Möglichkeiten gegeben hätte, wüssten wir zwar alles über Kutschen, Fahrwege, Logistik usw., aber das Auto wäre durch all dieses Wissen noch nicht erfunden" schreiben die Autoren. Ein provokanter Denkanstoß, dessen Schlussfolgerungen sicher nicht alle Leser teilen werden. Die Redaktion würde sich über kompetente Gegenrede sehr freuen, denn eines ist sicher, das Thema ist wichtig und die Auseinandersetzung damit unerlässlich.

Ich hoffe, bald von Ihnen zu hören. Bis bald und herzliche Grüße

\author{
Peter Pagel \\ Chefredakteur
}

https://doi.org/10.1007/s00287-018-1116-z 